Pacific Journal of Mathematics

TRANSFER IN GENERALIZED RESTACK COHOMOLOGY 


\title{
TRANSFER IN GENERALIZED PRESTACK COHOMOLOGY
}

\author{
Robert PIACENZA
}

The purpose of this paper is to define cohomology with coefficients in a local system of simplicial spectra and show this leads to a simple definition of the transfer homomorphism for a finite covering map. We wish to point out that the method of constructing the transer map in $\S 3$ of this paper gives a different approach to the one developed by $F$. W. Roush in his thesis.

1. Preliminaries. In this section we define those categories and functors of special interest. We assume the reader is familiar with the theory of simplicial sets and spectra as presented in [7], [9], [8], [4], and [3].

We let $\mathscr{S}\left(\mathscr{S}_{*}\right)$ be the category of simplicial sets (pointed simplicial sets) equipped with its standard closed model structure. Similarly we let $\mathscr{S p}(\mathscr{G} \mathscr{S} / p)$ be the category of simplicial spectra (group spectra) equipped with its standard closed model structure as defined in [3, Thm. 5, Pg. 439].

For $X \in \mathscr{S}(E \in \mathscr{S p})$ we let $X_{p}\left(E_{p}\right)$ be the set of $p$-simplexes of $X$ (stable $p$-simplexes of $E$ ).

As explained in [8, 4.4, Pg. 468] for each integer $q$ there is a functor $(q): \mathscr{S p}_{p} \rightarrow \mathscr{S}_{*}$ where $\{E(q)\}$ is the prespectrum associated to the spec-

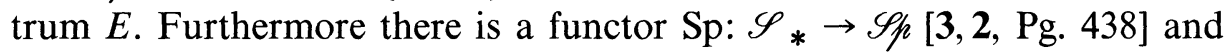
adjunction isomorphism

$$
\mathscr{S}_{*}(X, E(0)) \simeq \mathscr{S p}(\operatorname{Sp} X, E) .
$$

We let $c \mathscr{S p}(c \mathscr{G} \mathscr{S} / p)$ be the category of cosimplicial (group) spectra as defined in [2, Chap. X, §2, Pg. 267]. Thus for each $\mathbf{X} \in c \mathscr{S p}$ there is a spectrum $\mathbf{X}^{p}$ with coface operators

$$
\delta_{i}: \mathbf{X}^{p} \rightarrow \mathbf{X}^{p+1}
$$

and codegeneracies

$$
\sigma_{j}: \mathbf{X}^{p} \rightarrow \mathbf{X}^{p-1}
$$

satisfying the cosimplicial identities. 
1.1. Example. Let $X \in \mathscr{S}$ and $E \in \mathscr{S}$. Define $\mathbf{X}=\operatorname{Cs}(X ; E) \in c \mathscr{S p}$ by $\mathbf{X}^{p}=\operatorname{Set}\left(X_{p}, E\right)$ with coface and codegeneracies defined in the obvious way. [3, Appendix B, Pg. 457.]

Following Brown in [3, Appendix B] we define $T(\mathbf{X}) \in \mathscr{S p}$ for $X \in c \mathscr{S p}$ as follows: An $n$-simplex of $T(\mathbf{X})$ is a sequence $\left\{\psi_{p+n}^{p}\right\}_{p \geq 0}, \psi_{p+n}^{p} \in \mathbf{X}_{p+n}^{p}$, such that

(a)

$$
\begin{array}{rlrl}
d_{i} \psi_{p+n}^{p} & =\delta_{i} \psi_{p+n-1}^{p-1}, & & 0 \leq i \leq p, \\
s_{i} \psi_{p+n}^{p}=\sigma_{i} \psi_{p+n+1}^{p+1}, & & 0 \leq i \leq p .
\end{array}
$$

(b) $\left\{\psi_{p+n}^{p}\right\}$ has only finitely many nontrivial faces, these being defined by $d_{i}\left\{\psi_{p+n}^{p}\right\}=\left\{d_{i+p+1} \psi_{p+n}^{p}\right\}$.

We define approximations $T_{k}(\mathbf{X})$ to $T(\mathbf{X})$ by using finite sequences $\left\{\psi_{p+n}^{p}\right\}_{p \leq k}$ and we have $T(\mathbf{X})=\lim _{\leftarrow} T_{k}(\mathbf{X})$ using the natural projections $T_{k}(\mathbf{X}) \rightarrow T_{k-1}(\mathbf{X})$.

We define a fibration in $c \mathscr{S p}$ (weak equivalence in $c \mathscr{S p}$ ) just as in [2, 4.6, Pg. 275] ([2, 4.1, Pg. 273]).

The following proposition is proved in the same way as $[2,4.9, \mathrm{Pg}$. 276].

1.2. Proposition. (1) Every epimorphism $f: \mathbf{X} \rightarrow \mathbf{Y}$ in $c \mathscr{G} \operatorname{s}$ ph is a fibration.

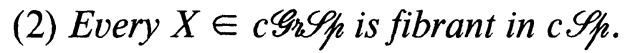

1.3. Corollary. If $f: \mathbf{X} \rightarrow \mathbf{Y}$ is an epimorphism (weak equivalence) in cGhSp then $T(f)$ is a fibration (weak equivalence) in $\mathscr{G}_{\mathrm{h}} \mathscr{S p}_{\mathrm{p}}$.

Proof. This follows from [3, Remarks, Pg. 435] or may be proved directly.

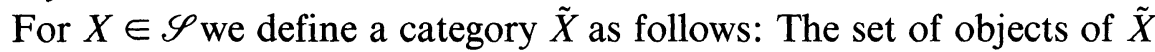
is $\amalg_{p \geq 0} X_{p}$, and for each face map $\partial_{l}$ or degeneracy $s_{j}$ we have a morphism $x \rightarrow \partial_{i} x, x \rightarrow s_{j} x$. We let these morphisms generate the morphisms of $\tilde{X}$ subject only to the simplicial identities. Compare with [5, Chapter I, Pg. 105]. By [7, 2.2, Pg. 24] each morphism $\theta: x \rightarrow \theta s$ can be uniquely expressed $\theta=s_{j_{1}} \cdots s_{J_{t}} \partial_{i_{1}} \cdots \partial_{i_{s}}$ for suitable face and degeneracy operators.

We define the category of prestacks of spectra (group spectra) over $X$

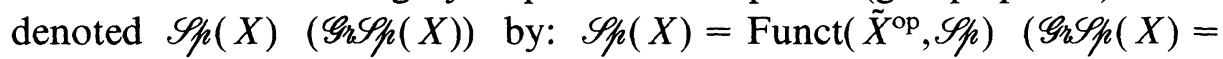
$\left.\operatorname{Funct}\left(X^{\mathrm{op}}, \mathscr{G}_{h} \mathscr{P}_{h}\right)\right)$. 
A morphism $f: \mathscr{E} \rightarrow \mathscr{F}$ of $\mathscr{S p}(X)$ is called a fibration (weak equivalence) if $f(x): \mathscr{E}(x) \rightarrow \mathscr{F}(x)$ is a fibration (weak equivalence) in $\mathscr{S p}$ for each simplex $x$ of $X . \mathscr{E}$ is called a Kan prestack if the unique morphism $\mathscr{E} \rightarrow *$ to the trivial prestack is a fibration.

1.4. REMARK. The category of Kan prestacks is a category of fibrant objects in the sense of [3, Part I, Pg. 420].

A prestack $\mathscr{E}$ is called a stack of spectra if $\mathscr{E}(\theta)$ is an isomorphism for each morphism $\theta$ in $\tilde{X}$.

1.5. EXAMPLE. For $E \in \mathscr{S p}$ we define the constant stack $\mathscr{E}$ with value $E$ by: $\mathscr{E}(x)=E$ and $\mathscr{E}(\theta)=1_{E}$ for any morphism $\theta$.

We leave the proof of the next proposition to the reader.

1.6. Proposition. Every stack $\mathscr{E}$ over $\Delta[p]$, the standard p-simplex is isomorphic to the constant stack with value $\mathscr{E}\left(\Delta_{p}\right)$ where $\Delta_{p}$ is the unique nondegenerate p-simplex of $\Delta[p]$.

For $g: X \rightarrow Y$ a morphism in $\mathscr{S}$ we let $\tilde{g}: \tilde{X} \rightarrow \tilde{Y}$ be the obvious induced functor. We define the pullback functor $g^{\#}: \mathscr{S p}_{h}(Y) \rightarrow \mathscr{S p}(X)$ by

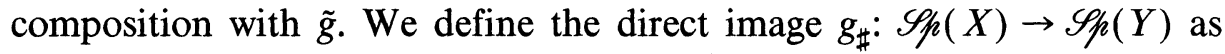
follows: For $\mathscr{E} \in \mathscr{S p}(X)$ define $\mathscr{F}=g_{\sharp} \mathscr{E} \in \mathscr{S p}(Y)$ by setting $\mathscr{F}(y)=$ $\Pi_{x \in g^{-1}(y)} \mathscr{E}(x) . \mathscr{F}$ is defined on morphisms of $Y$ in the obvious way. One easily shows $g_{\sharp}$ is right adjoint to $g^{\#}$. Compare with [5, Chapter I, §2].

2. Generalized prestack cohomology. In this section we define and study cohomology with coefficients in a prestack of spectra.

Throughout this section $\mathscr{G}$ is a fixed prestack of group spectra over $X$, i.e., $\mathscr{G} \in \operatorname{Gn} \mathscr{S p}(X)$. Generalizing Example 1.1 we define a cosimplicial group spectrum denoted $\mathbf{X}=\operatorname{Cs}(X ; \mathscr{G})$ as follows: For $p \geq 0$ let $\mathbf{X}^{p}=$ $\prod_{x \in X_{p}} \mathscr{G}(x)$. We view $\mathbf{X}^{p}$ as a group spectrum of functions $\psi: X_{p} \rightarrow \bigcup \mathscr{G}(x)$. If $\partial_{i}$ is a face map of $x$ then for each $y \in X_{p+1}$ we have a morphism $y \rightarrow \partial_{i} y=x$ of $\tilde{X}$ and thus we may define a coface operator $\delta_{i}: \mathbf{X}^{p} \rightarrow \mathbf{X}^{p+1}$ by:

$$
\delta_{i} \psi(y)=\mathscr{G}\left(\partial_{i}\right)(\psi(x))
$$

Similarly for the codegeneracies $\sigma_{j}: \mathbf{X}^{p+1} \rightarrow \mathbf{X}^{p}$. For each integer $q$ we define the cohomology of $X$ with coefficients $\mathscr{G}$ denoted $H^{q}(X ; \mathscr{G})$ by 
setting

$$
H^{q}(X ; \mathscr{G})=\pi_{-q} T(\mathbf{X})
$$

where $\pi_{*}$ is the stable homotopy group functor.

To emphasize the coefficients we will sometimes write $T(\mathbf{X})=$ $T(X ; \mathscr{G})$ and $T_{k}(X ; \mathscr{G})$ for the approximations.

A morphism $f: \mathscr{G}_{1} \rightarrow \mathscr{G}_{2}$ in $\mathscr{G}_{h} \mathscr{P}(X)$ clearly induces a morphism

$$
f^{\prime}: \operatorname{Cs}\left(X ; \mathscr{G}_{1}\right) \rightarrow \operatorname{Cs}\left(X ; \mathscr{G}_{2}\right)
$$

and thus an induced coefficient homomorphism

$$
f_{*}: H^{*}\left(X ; \mathscr{G}_{1}\right) \rightarrow H^{*}\left(X ; \mathscr{G}_{2}\right) .
$$

It follows from 1.3 that if $f$ is a weak equivalence then $f_{*}$ is an isomorphism. We may thus extend the definition of cohomology to coefficients $\mathscr{E} \in \mathscr{S p}(X)$ by setting

$$
H^{*}(X ; \mathscr{E})=H^{*}(X ; F \mathscr{E})
$$

where $F \mathscr{E}$ is the composition of $\mathscr{E}$ with the free group spectrum defined in $[8,5.2$, pg. 470].

2.1. EXAMPLE. If $\mathscr{E} \in \mathscr{S} p(X)$ is the constant stack with value $E \in \mathscr{S} p$ then $T(X ; \mathscr{E})=\mathscr{H}$ om $(X ; F E)$ as defined in [3, Appendix B]. Thus $H^{*}(X ; \mathscr{E})=H^{*}(X ; E)$ is the generalized singular cohomology with coefficients $E$.

To define the relative cohomology for the pair $(X, A)$ we proceed as follows: Let $i: A \rightarrow X$ be the inclusion and $\mathscr{G}^{A}=i_{\sharp} i^{\#} \mathscr{G}$. We thus obtain a short exact sequence,

$$
\mathscr{G}^{A} \hookrightarrow \stackrel{f}{\rightarrow} \mathscr{G}_{A} \quad \text { in } \operatorname{Gr} \mathscr{S p}(X) .
$$

We define $H^{*}(X, A ; \mathscr{G})=H^{*}\left(X ; \mathscr{G}_{A}\right)$ and obtain the natural long exact sequence from the epimorphism

$$
f^{\prime}: \operatorname{Cs}(X ; \mathscr{G}) \rightarrow \operatorname{Cs}\left(X ; \mathscr{G}_{A}\right) \quad \text { induced by } f .
$$

Let $g: X \rightarrow Y$ be a map in $\mathscr{S}$ and $\mathscr{F} \in \mathscr{G} \mathscr{S p}(Y)$. There is an induced morphism $\check{g}: \operatorname{Cs}(Y ; \mathscr{F}) \rightarrow \operatorname{Cs}\left(X ; g^{\#} \mathscr{F}\right)$ which is defined as follows: Let $\psi$ : $Y_{p} \rightarrow \bigcup \mathscr{F}(y)$. For each $x \in X_{p}$ let $\check{g} \psi(x)=\psi(g(x))$. One easily checks $\check{g}$ is a map of cosimplicial group spectra. We let $g^{*}: H^{*}(Y ; \mathscr{F}) \rightarrow$ $H^{*}\left(X ; g^{\# \mathscr{F}}\right)$ be the map induced by $\check{g}$.

Now let $\mathscr{G} \in \operatorname{Gn} \mathscr{S p}(X)$ and define $\check{g}: \operatorname{Cs}\left(Y ; g_{\sharp} \mathscr{G}\right) \rightarrow \operatorname{Cs}(X ; \mathscr{G})$ as follows: Let $\psi: Y_{p} \rightarrow \bigcup g_{\sharp} \mathscr{G}(y)$. Then for $z \in X_{p}$ with $f(z)=y$ we let 
$\hat{g} \psi(z)=p_{z} \psi(g(z))$ where $p_{z}: \prod_{x \in g^{-1}(y)} \mathscr{G}(x) \rightarrow \mathscr{G}(z)$ is the natural projection onto $\mathscr{G}(z)$. One easily checks that $\hat{g}$ is an isomorphism of cosimplicial group spectra. Thus the induced map

$$
g^{*}: H^{*}\left(Y ; g_{\sharp} \mathscr{G}\right) \rightarrow H^{*}(X ; \mathscr{G})
$$

is an isomorphism.

We define the Atiyah-Hirzebruch spectral sequence of $\mathscr{G}$ to be the stable Bousfield-Kan spectral sequence of the tower $\left\{T_{k}(X ; \mathscr{G})\right\}[3$, Appendix B]. This spectral sequence converges to $H^{*}(X ; \mathscr{G})$ if $X$ is finite dimensional or if $\pi_{q}(\mathscr{G})=0$ for all suffficiently large $q . \pi_{*}(\mathscr{G})$ is the composition of $\mathscr{G}$ with the stable homotopy group functor $\pi_{*}$. The following results is an easy application of the convergence of this spectral sequence.

2.2. Proposition. Let $\mathscr{E} \in \mathscr{S p}(X)$ and suppose

$$
\pi_{n}(\mathscr{E})= \begin{cases}0 & n \neq q \\ \mathscr{L} & n=q .\end{cases}
$$

Then $H^{p}(X ; \mathscr{E}) \simeq H^{p+q}(X ; \mathscr{L})$ where the right side is the prestack cohomology of $\mathscr{L}$ as defined in [6].

We mention one other example before going on.

2.3. EXAMPLE. Equivariant generalized cohomology. Let $G$ be a group and $J$ the category of canonical $G$ orbits as in [11]. Let $E: J^{\text {op }} \rightarrow \mathscr{G} \mathscr{C P p}_{h}$ be a system of coefficients. For $X$ a $G$ simplicial set, i.e., $X \in G-S S$ we define $H_{G}^{q}(X ; E)=\pi_{-q} \mathscr{H}_{J}(X, E)$ where we view $X \in J S$ as in [11, III]. Define a stack $\mathscr{E}$ over $X / G$ by setting for $x \in X / G$

$$
\mathscr{E}(x)=\operatorname{Nat}\left(p^{-1}(x), E\right)
$$

where $p: X \rightarrow X / G$ is the natural projection. One may easily check that $\mathscr{H C m}_{J}(X, E) \simeq T(X / G, \mathscr{E})$ and thus $H_{G}^{*}(X ; E) \simeq H^{*}(X / G ; \mathscr{E})$. Compare this with [11, Thm. 2.6] and [11, Thm. 3.11].

We let $\mathscr{S} \downarrow X$ be the category of simplicial sets over $X$ as in [5, Chapter III]. For $t: Z \rightarrow X$ in $\mathscr{S} \downarrow X$ we define $H^{*}(t ; \mathscr{G})=H^{*}\left(Z ; t^{\sharp} \mathscr{G}\right)$ with a similar definition for morphisms of $\mathscr{S} \downarrow X$. We briefly sketch a representation theorem for $H^{*}(; \mathscr{G})$ leaving the details to the reader. For the remainder of this section we shall assume $\mathscr{G}$ is a stack.

First we introduce the following notation. For a spectrum $E$ and integer $q$ we let $E\langle q\rangle=E(q)_{0}$; i.e., the set of 0-simplexes of the simplicial set $E(q)$. 
We define a simplicial set $K(q, \mathscr{G})$ as follows: Let

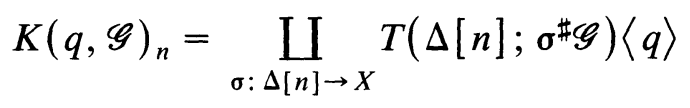

and for each map

$$
\Delta[p] \underset{\lambda_{\searrow X \swarrow^{\sigma}}}{\stackrel{g}{\rightarrow}} \Delta[n] \text { of } \mathscr{S} \downarrow X
$$

let $\bar{g}: K(q, \mathscr{G})_{n} \rightarrow K(q, \mathscr{G})_{p}$ be the obvious induced map. Clearly $K(q, \mathscr{G})$ is a simplicial set with the obvious projection

$$
\pi_{q}: K(q, \mathscr{G}) \rightarrow X
$$

We note that $\pi_{q}$ is a group object in $\mathscr{S} \downarrow X$.

Given $\psi \in T(X ; \mathscr{G})\langle q\rangle$ we define a section $\psi^{\prime}$ of $\pi_{q}$ as follows: For $x=\sigma\left(\Delta_{n}\right) \in X_{n}$ let $\psi^{\prime}(x)=\sigma^{*}(\psi) \in T\left(\Delta[n], \sigma^{\sharp} \mathscr{G}\right)\langle q\rangle$. The proof of the following proposition is left to the reader.

2.4. Proposition. The above correspondence $\psi \mapsto \psi^{\prime}$ is an isomorphism of groups. Furthermore the sections $\psi_{1}^{\prime}$ and $\psi_{2}^{\prime}$ are fiber homotopic over $X$ (as defined in [6, §2, Pg. 602]) iff $\psi_{1}$ and $\psi_{2}$ are homotopic 0-simplexes of $T(X ; \mathscr{G})\langle q\rangle$.

Since the above isomorphism respects pullbacks we have the following theorem.

2.5. THEOREM. There is a natural isomorphism of groups $H^{q}(t ; \mathscr{G}) \simeq$ $\left[t, \pi_{q}\right]_{X}$ where the right side is the group of fiber homotopy classes of maps.

Compare this with [6, Thm. A, Pg. 603] and [10, 5.5, Pg. 342].

Since $\mathscr{G}$ is a stack we may use 1.6 to show $\pi_{q}$ is a fibre bundle in the sense of [9, Def. 11.8, Pg. 45]. The following proposition is proved just as [10, 2.6, Pg. 337].

2.6. Proposition. Let

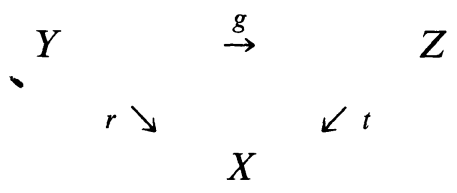

be a map in $\mathscr{S} \downarrow X$. If $g$ is an ordinary homotopy equivalence in $\mathscr{S}$ then $g^{*}$ : $H^{*}(t ; \mathscr{G}) \rightarrow H^{*}(r ; \mathscr{G})$ is an isomorphism. 
Compare 2.6 with [7, 4.8, Pg. 161] and with [10, 5.2, Pg. 341].

3. The transfer for prestack cohomology. In this section we define the transfer and state its most important properties.

Let $F$ be a finite set viewed as a trivial simplicial set; i.e., all the face and degeneracies are the identity map. A map $g: X \rightarrow Y$ in $\mathscr{S}$ is called a finite covering map with fibre $F$ if $g$ is a fibre bundle with fibre $F$ in the sense of [8, Def. 11.8, Pg. 45]. This is equivalent to the definition found in [7, Appendix One].

If $B \subseteq Y$ and $A=g^{-1}(B)$ then we call $g:(X, A) \rightarrow(Y, B)$ a finite covering map of pairs.

Let $\mathscr{E}$ be a fixed prestack in $\mathscr{S p}(Y)$.

Then we have the following.

3.1. EXISTENCE THEOREM. For each finite covering map g there exists an abelian group homomorphism $g^{!}: H^{*}\left(X ; g^{\#} \mathscr{E}\right) \rightarrow H^{*}(Y ; \mathscr{E})$ called transfer in $\mathscr{E}$ cohomology with the following properties:

3.2. Transfer is functorial over pullbacks of coverings.

3.3. Transfer for the identity covering is the identity.

3.4. If a covering splits as a disjoint union of coverings then transfer over the total covering is the sum of the transfers over the component coverings.

3.5. Transfer for a composition of coverings is the composition of the transfers.

3.6. Transfer induces a morphism on the $E_{2}$ term of the Atiyah-Hirzebruch spectral sequence and is compatible with the differentials of that sequence.

3.7. If $g$ is a finite covering map of pairs there is a transfer $g$ !: $H^{*}(X, A ; \mathscr{E}) \rightarrow H^{*}(Y, B ; \mathscr{E})$ that agrees with our previous transfer if $B$ is empty. Furthermore the transfer is compatible with the coboundary operator of a pair.

3.8. Transfer is compatible with the coboundary operator of suitably defined Mayer-Vietoris sequences.

Proof. To define the transfer consider the prestack $\mathscr{E}^{1}=g_{\sharp} g^{\sharp} \mathscr{E}$. There is a subprestack $\mathscr{E}^{2}$ of $\mathscr{E}^{1}$ with $\mathscr{E}^{2}(y)=V \mathscr{E}(y)$; i.e., a coproduct of card $\left(g^{-1}(y)\right)$ copies of $\mathscr{E}(y)$. The natural inclusion $i: \mathscr{E}^{2} \rightarrow \mathscr{E}^{1}$ is a weak equivalence in $\mathscr{S p}(Y)$. Let $s: \mathscr{E}^{2} \rightarrow \mathscr{E}$ be the natural map; i.e., for each $y$, $s_{y}: \mathscr{E}^{2}(y) \rightarrow \mathscr{E}(y)$ is a coproduct of identities. Thus we have the diagram: $\mathscr{E}^{1} \leftarrow \mathscr{E}^{2} \stackrel{s}{\rightarrow} \mathscr{E}$ and in chomology we obtain

$$
H^{*}\left(X ; g^{\sharp} \mathscr{E}\right) \simeq H^{*}\left(Y ; \mathscr{E}^{1}\right) \simeq H^{*}\left(Y, \mathscr{E}^{2}\right) \stackrel{s_{*}}{\rightarrow} H^{*}\left(Y ; \mathscr{E}^{\mathscr{N}}\right) .
$$


We use this last diagram to define the transfer $g$ ! in the obvious way. One may easily check properties 3.2 through 3.8 using suitable properties of prestack cohomology. The details are left to the reader.

The following corollary is an immediate consequence of the naturality of the transfer $g$ !

3.9. Corollary. For each finite covering map $g: X \rightarrow Y$ there is induced a natural stable map

$$
\operatorname{st}(g): \operatorname{Sp}\left(Y^{+}\right) \rightarrow \operatorname{Sp}\left(X^{+}\right) .
$$

Furthermore $\operatorname{st}(g)$ induces $g^{!}$in $\mathscr{E}$ cohomology if $\mathscr{E}$ is a constant stack in $\operatorname{Sp}(Y)$.

3.10. REMARK. (1) The stable map st $(g)$ may be used to construct the transfer in generalized homology.

(2) F. W. Roush in his thesis [13] constructs the transfer by geometrical methods. For a description of this approach see [1, Chapter 4]. It follows from the uniqueness theorem [13, Thm. 1B, Pg. 92] that transfer in stack cohomology agrees with the transfer as defined by Roush in [13].

(3) The construction of transfer here parallels that given for generalized sheaf cohomology in [12].

(4) Clearly a version of [12, Prop. 3.7] may be proved for stack cohomology.

\section{REFERENCES}

[1] J. F. Adams, Infinite loop spaces, Annals of Math. studies number 90, Princeton, 1978.

[2] A. K. Bousfield and D. M. Kan, Homotopy Limits Completions and Localizations, Springer lecture notes number 304. 1972.

[3] K. Brown, Abstract homotopy theory and generalized sheaf cohomology, Trans. Amer. Math. Soc., 186 (1973), 419-458.

[4] D. Burghelia and A. Deleanu, The homotopy category of spectra, Illinois J. Math., 11 (1967), 454-473.

[5] Y.-C. Chen, Stacks, costacks and axiomatic homology, Trans. Amer. Math. Soc., 145 (1969), 105-116.

[6] , On Spanier's higher order operations, Proc. Amer. Math. Soc., 31 (1972), 601-604.

[7] P. Gabriel and M. Zisman, Calculus of Fractions and Homotopy Theory, SpringerVerlag, 1967.

[8] D. Kan, Semisimplicial spectra, Illinois J. Math., 7 (1963), 463-478.

[9] P. May, Simplicial Objects in Algebraic Topology, Van Nostrand, 1967.

[10] R. J. Piacenza, Cohomology of fiber spaces is representable, Illinois J. Math., 23 (1979), 334-343. 
[11] Cohomology of diagrams and equivariant singular theory, Pacific J. Math., 91 (1981), 435-444.

[12] $\quad$ Transfer in generalized sheaf cohomology, Proc. Amer. Math. Soc., 90 (1984), 653-656.

[13] F. W. Roush, Transfer in generalized cohomology theories, Thesis, Princeton, 1971.

Received May 10, 1983.

UNIVERSITY OF ALASKA

FAIRBANKS, AK 99701 



\section{PACIFIC JOURNAL OF MATHEMATICS \\ EDITORS}

Donald BABBITT (Managing Editor)

University of California

Los Angeles, CA 90024

J. Dugundu

University of Southern Californa

Los Angeles, CA 90089-1113

R. FINN

Stanford University

Stanford, CA 94305

HermanN FLaSChKa

University of Arizona

Tucson, AZ 85721

\author{
C. C. MOORE \\ University of California \\ Berkeley, CA 94720 \\ ARTHur Ogus \\ University of California \\ Berkeley, CA 94720 \\ Hugo Rossi \\ University of Utah \\ Salt Lake City, UT 84112 \\ H. SAMELSON \\ Stanford University \\ Stanford, CA 94305
}

ASSOCIATE EDITORS
R. ARENS
E. F. BECKENBACH
B. H. NeUmanN
F. WOLF
K. YOSHIDA (1906-1982)

\section{SUPPORTING INSTITUTIONS}

UNIVERSITY OF ARIZONA

UNIVERSITY OF BRITISH COLUMBIA

UNIVERSITY OF OREGON

CALIFORNIA INSTITUTE OF TECHNOLOGY

UNIVERSITY OF CALIFORNIA

MONTANA STATE UNIVERSITY

UNIVERSITY OF SOUTHERN CALIFORNIA

UNIVERSITY OF NEVADA, RENO

STANFORD UNIVERSITY

UNIVERSITY OF HAWAII

NEW MEXICO STATE UNIVERSITY

UNIVERSITY OF TOKYO

UNIVERSITY OF UTAH

WASHINGTON STATE UNIVERSITY

OREGON STATE UNIVERSITY

UNIVERSITY OF WASHINGTON 


\section{Pacific Journal of Mathematics}

\section{Vol. 116, No. $1 \quad$ November, 1985}

K. Adachi, Le problème de Lévi pour les fibrés grassmanniens et les variétés

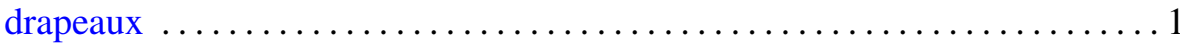

John MacLeod Ball, Remarks on the paper: "Basic calculus of variations" . . . 7 John Kelly Beem and Phillip E. Parker, Whitney stability of solvability . . . 11 Alberto Facchini, Decompositions of algebraically compact modules .......25

S. S. Khare, Finite group action and equivariant bordism $\ldots \ldots \ldots \ldots . \ldots 39$

Horst Leptin, A new kind of eigenfunction expansions on groups $\ldots \ldots \ldots . .45$

Pei-Kee Lin, Unconditional bases and fixed points of nonexpansive

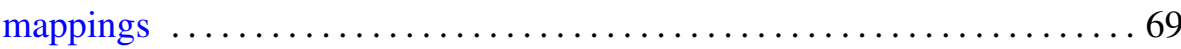

Charles Livingston, Stably irreducible surfaces in $S^{4} \ldots \ldots \ldots \ldots \ldots 77$

Kevin Mor McCrimmon, Nonassociative algebras with scalar involution . . .885

Albert Milani, Singular limits of quasilinear hyperbolic systems in a

bounded domain of $\mathbf{R}^{3}$ with applications to Maxwell's equations

Takemi Mizokami, On $M$-structures and strongly regularly stratifiable

spaces

Jesper M. Møller, On the homology of spaces of sections of complex

projective bundles

Nikolaos S. Papageorgiou, Carathéodory convex integrand operators and

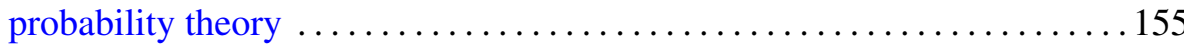

Robert John Piacenza, Transfer in generalized prestack cohomology 185

Lance W. Small and Adrian R. Wadsworth, Integrality of subrings of matrix rings ...

James Michael Wilson, On the atomic decomposition for Hardy spaces 